\title{
Clinical Progress on Management of Pneumonia Due to COVID-19 With Chinese Traditional Patent Medicines
}

\author{
Ying Wu and Ping Zhong* \\ Department of Neurology, Shanghai Shidong Hospital of Yangpu District, Shanghai, China
}

Background: The outbreak of new coronavirus has tremendously threatened the public health system worldwide, including China. Chinese patent medicines (CPMs) have greatly contributed to the prevention and treatment of this viral infection, as well as the recovery of patients with COVID-19 infection. Therefore, numerous experts and guidelines recommend to take CPMs to treat pneumonia due to COVID-19.

Aim of the Study: The present study reviewed CPMs recommended by the < Guidelines for diagnosis and management of COVID-19 ( $8^{\text {th }}$ edition) $>$ regarding evidence of their efficacy from clinical studies and the underlying mechanisms, which will lay the foundation for clinical use of these CPMs for COVID-19.

OPEN ACCESS

Edited by:

Maria De Lourdes Pereira, University of Aveiro, Portugal

Reviewed by:

Mahaveer Dhobi,

Delhi Pharmaceutical Sciences and

Research University, India

Sunil Kayesth,

University of Delhi, India

*Correspondence:

Ping Zhong zphg/@163.com

Specialty section:

This article was submitted to

Ethnopharmacology,

a section of the journal

Frontiers in Pharmacology

Received: 18 January 2021

Accepted: 19 August 2021

Published: 02 September 2021

Citation:

Wu Y and Zhong P (2021) Clinical

Progress on Management of

Pneumonia Due to COVID-19 With

Chinese Traditional Patent Medicines.

Front. Pharmacol. 12:655063.

doi: 10.3389/fphar.2021.655063
Methods: The composition, efficacy, indications, history of use, and relevant clinical research on 14 recommended CPMs, including Huoxiangzhengqi capsules (pills, liquid, oral solution), Jinhuaqinggan granules, Lianhuaqingwen capsules (granules), Shufengjiedu capsules, Xiyanping injections, Xuebijing injections, Reduning injections, Tanreqing injections, Xingnaojing injections, Shenfu injections, Shengmai injections, Angongniuhuang pills, Suhexiang pills, were searched in both Chinese and English databases based on differences in stages of the disease and manifestations of such patients. Advantages of these CPMs over conventional treatments and their underlying mechanisms were explored by analyzing results from published articles and undergoing clinical trials.

Results: Findings from clinical studies and Chinese experience in using these CPMs showed that CPMs, when used in combination with conventional treatments, were effective in managing COVID-19 with few side effects.

Conclusion: CPMs have excellent efficacy in managing COVID-19 with a great potential for clinical use.

Keywords: COVID-19, Chinese patent medicines, clinical studies, traditional Chinese medicine, pharmacological evaluation

Abbreviations: ACE2, angiotensin converting enzyme II; 3CLpro, 3C-like protease; CAMSAP, calmodulin-regulated spectrinassociated protein; $\mathrm{CI}$, confidence interval; $\mathrm{COPD}$, chronic obstructive pulmonary diseases; $\mathrm{CPM}$, Chinese patent medicine; CT, computed tomography; HSP, heat shock protein; IC, inhibitory concentration; ICH, intracerebral hemorrhage; IL, interleukin; LPS, Lipopolysaccharide; MAPK, mitogen-activated protein kinases; NF-Kb, nuclear factor kappa B; OR, odds ratio; PTGS, prostaglandin-endoperoxide synthase; RCT, randomized controlled trial; RR, relative risk; SARS-CoV-2, severe acute respiratory syndrome coronavirus 2; TCM, traditional chinese medicine; TNF, tumor necrosis factor alpha; WMD, weighted mean difference. 


\section{INTRODUCTION}

COVID-19 broke out in Wuhan, Hubei Province of China and spread to other regions in China since December 2019. In the following months, it has spread to other countries and become a global public health threat (The Lancet, 2020). It was nearly under control in China after taking proactive measures by the Chinese government and relevant authorities in the first few months. Chinese traditional patent medicines (CPMs) have been widely used based on the diagnosis according to the Traditional Chinese Medicine (TCM) theory and they showed superiority to general treatments. This reflects the advantages of TCM in fighting against COVID-19. TCM is not only helpful for patients with different symptoms but also for those at different stages of the disease. It is recommended to halt or slow the progression of this disease in either the observation period or when symptoms manifest (Chen et al., 2020b; Shao et al., 2020). An official report has shown that $91.5 \%$ of patients with COVID-19 have used TCM therapies in China and the efficacy is over 90\% (http:// www.gov.cn/). The present study aimed to review relevant clinical studies, underlying mechanisms, and clinical efficacy of 14 CPMs recommended by National Health Commission of People's Republic of China in < Guidelines for diagnosis and management of COVID-19 $\left(8^{\text {th }}\right.$ edition) $>$. This will facilitate the active application of these CPMs to patients with COVID-19 in other countries with an aim to control this plague.

\section{CHINESE TRADITIONAL PATENT MEDICINES USED FOR COVID-19 TREATMENT AND RELEVANT CLINICAL STUDIES}

In $<$ Guidelines for diagnosis and management of COVID-19 $\left(8^{\text {th }}\right.$ edition) $>, 14$ CPMs were recommended with six of them being of oral dosage form and eight of injection dosage form (Table 1; Table 2). For suspected COVID-19 patients or those who had close contact with these patients, medical observation was required. If they showed symptoms like fatigue, gastrointestinal upset, or fever, 4 CPMs of oral dosage form were recommended and these included: Huoxiangzhengqi capsules for those with gastrointestinal symptoms, Jinhuaqinggan granules, Lianhuaqingwen capsules or granules, and Shufengjiedu capsules or granules for those with fever. Patients with severe symptoms were classified into two categories: Yidubifei (literal meaning: functions of the lungs were compromised due to viral infection. Patients showed symptoms of fever, face flushing, coughing with little thick yellowish sputum, or sputum with blood, shortness of breath, fatigue, dry mouth with sticky saliva, loss of appetite, difficulty in defecating, small volume of dark urine, red tongue, yellowish and greasy coating of the tongue, rapid pulsation) and Qiyingliangfan (a syndrome characterized by high fever and thirst, shortness of breath, delirium and loss of consciousness, blurred vision, maculae on the skin, hematemesis, epistaxis, seizures, red tongue with little or no coating, sunken, fine, and rapid pulsation, or superficial, large, and rapid pulsation). CPMs in the injection dosage form were recommended for these patients who were admitted due to the severe symptoms, including Xiyanping injection, Xuebijing injection, Reduning injection, Tanreqing injection, Xingnaojing injection. Shenfu injection, Shengmai injection, Shenmai injection were also recommended to use in combination with the abovementioned injection dosage forms for patients with breathing difficulty, disturbance in consciousness, and mechanical ventilation. These injections could be used alone if they are similar to each other in their therapeutic mechanism or two of them used in combination based on the condition of patients. These injections can also be used in combination with decoctions. For patients with severe symptoms, two oral dosage forms were also recommended, including Angongniuhuang and Suhexiang pills (Figure 1).

CPMs are generally comprised of multiple herbs. For example, Suhexiang pills are comprised of 15 herbs, but Xiyanping injection is the only one that has one effective compound. Some herbs have the same compounds. For example, Jinhuaqinggan granules, Lianhuaqingwen granules, Reduning injection, and Tanreqing injection have the same herbLonicera japonica Thunb. (Jinyinhua). Lianhuaqingwen capsules (granules), Shufengjiedu capsules, and Tanreqing injection have the same herb-Forsythia suspensa (Thunb.) Vahl (Lianqiao) (Figure 2).

Both Chinese and English databases were searched for relevant articles regarding the 14 recommended CPMs. These databases included China National Knowledge Infrastructure (CNKI), Wanfang database, Sinomed Database, PubMed and Embase. The time was limited to the beginning of the database to December 2, 2020. Multiple keywords for COVID-19 were used, including COVID-19, 2019 novel coronavirus, SARSCoV-2, 2019-nCoV, coronavirus disease 2019, coronavirus disease-19. Key words for CPMs included its general names and commercial names.

By 20:00 December 2, 2020, the Chinese Clinical Trial Registry website www.chictr.org.cn/ showed 15 ongoing clinical trials on CPMs for COVID-19, including four trials on Xiyanping injection, two on Xuebijing injection, two on Reduning injection, one on Tanreqing injection, one on Shenfu injection, one on Jinhuaqing granules, and four on Lianhuaqingwen granules/capsules (Table 3).

\section{CHINESE TRADITIONAL PATENT MEDICINES USED IN THE OBSERVATION PERIOD}

Lianhuaqingwen granules are based on Maxingshigan decoction from $<$ Shanghanlun (Treatise on febrile caused by cold) $>$ and Yinqiaosan from $<$ Wenbingtiaobian (Differentiation on Febrile Diseases) $>$. The major therapeutic effects include opening the lungs as well as clearing the heat and toxins (Li H. R. et al., 2020). Based on findings from network pharmacology and molecular docking analyses, Lianhuaqingwen capsules fight against COVID-19 through multi-components, multi-targets, and multi-pathways. The major effective compounds of this herbal 
TABLE 1 | Summary of recommended oral CPMs in the Guidelines on Diagnosis and Management of COVID-19.

Drug name
Huoxiangzhengqi Capsule/Pill/ Oral liquids
Compositions

Pogostemon cablin (Blanco) Benth. Perilla frutescens (L.) Britton Angelica dahurica (Hoffm.) Benth. \& Hook.f. ex Franch. \& Sav.

Atractylodes macrocephala Koidz.

Citrus $\times$ aurantium L.

Pinellia ternata (Thunb.) Makino

Magnolia officinalis Rehder \& E.H.Wilson

Poria cocos (Schw. ) Wolf.

Platycodon grandiflorus (Jacq.) A.DC.

Glycyrrhiza uralensis Fisch. ex DC.

Areca catechu L.

Ziziphus jujuba Mill.

Zingiber officinale Roscoe

\begin{tabular}{|c|c|c|}
\hline \multirow[t]{12}{*}{ Jinhuaqinggan Granules } & Jinyinhua & Lonicera japonica Thunb. \\
\hline & Shigao & Gypsum Fibrosum \\
\hline & Mahuang & Ephedra sinica Stapf \\
\hline & Kuxingren & Prunus armeniaca L. \\
\hline & Huangqin & Scutellaria baicalensis Georgi \\
\hline & Lianqiao & Forsythia suspensa (Thunb.) Vahl \\
\hline & Zhebeimu & Fritillaria thunbergii Miq. \\
\hline & Zhimu & Anemarrhena asphodeloides Bunge \\
\hline & Niubangzi & Arctium lappa L. \\
\hline & Qinghao & Artemisia annua L. \\
\hline & Bohe & Mentha canadensis L. \\
\hline & Gancao & Glycyrrhiza uralensis Fisch. ex DC. \\
\hline \multirow{13}{*}{$\begin{array}{l}\text { Lianhuaqinwen Capsule/ } \\
\text { Granules }\end{array}$} & Lianqiao & Forsythia suspensa (Thunb.) Vahl \\
\hline & Jinyinhua & Lonicera japonica Thunb. \\
\hline & Mahuang & Ephedra sinica Stapf \\
\hline & Kuxingren & Prunus armeniaca L. \\
\hline & Shigao & Gypsum Fibrosum \\
\hline & Banlangen & Isatis tinctoria L. \\
\hline & Mianmaguanzhong & Dryopteris crassirhizoma Nakai \\
\hline & Yuxingcao & Houttuynia cordata Thunb. \\
\hline & Guanghuoxiang & Pogostemon cablin (Blanco) Benth. \\
\hline & Dahuang & Rheum officinale Baill. \\
\hline & Hongjingtian & Rhodiola rosea Linn. \\
\hline & Bohenao & Mentholum \\
\hline & Gancao & Glycyrrhiza uralensis Fisch. ex DC. \\
\hline \multirow{8}{*}{$\begin{array}{l}\text { Shufengjiedu Capsule/ } \\
\text { Granules }\end{array}$} & Huzhang & Reynoutria japonica Houtt. \\
\hline & Lianqiao & Forsythia suspensa (Thunb.) Vahl \\
\hline & Banlangen & Isatis tinctoria L. \\
\hline & Chaihu & Bupleurum chinense DC. \\
\hline & Baijiangcao & Patrinia scabiosifolia Link \\
\hline & Mabiancao & Verbena officinalis L. \\
\hline & Lugeng & $\begin{array}{l}\text { Phragmites australis (Cav.) Trin. ex } \\
\text { Steud. }\end{array}$ \\
\hline & Gancao & Glycyrrhiza uralensis Fisch. ex DC. \\
\hline \multirow[t]{11}{*}{ Angong Niuhuang Pill } & Niuhuang & Calculus Bovis \\
\hline & Shuiniujiao & Buffalo Horn Extract \\
\hline & Shexiang & Moschus \\
\hline & Zhenzhu & Margarita \\
\hline & Zhusha & Cinnabar \\
\hline & Xionghuang & Realgar \\
\hline & Huanglian & Coptis chinensis Franch. \\
\hline & Huangqin & Scutellaria baicalensis Georgi \\
\hline & Zhizi & Gardenia jasminoides J.Ellis \\
\hline & Yujin & Curcuma aromatica Salisb. \\
\hline & Bingpian & Borneolum Syntheticum \\
\hline
\end{tabular}

\section{Clinical indications}

Resolve dampness with aromatics and disperse cold as well as regulate the stomach, used for wind-cold, internal dampness stasis, or humidity induced viral infection, manifested as headache, lightheadedness, fullness of the chest, distension and pain in the belly, vomiting and diarrhea, compatible with Gl type of cold.

\begin{abstract}
Dispel the wind and ventilate the lungs, clear heat and toxin, used for fever, mild cold feeling, inflamed throat with pain, stuffy nose with nose discharge, thirst, coughing with/without sputum, red tongue, thin and yellow coat of the tongue, fast pulse due to external infection.
\end{abstract}

Clear heat and toxins, ventilatelungs and expel heat, used for syndromes of lung infection by influenza viruses, manifested as fever or high fever, cold feeling, sore muscles, stuffy nose with discharge, coughing, headache, dry and sore throat, reddish tongue, yellow or yellow thick coat of the tongue.
Dispel the wind and clear heat and toxins, pacify the throat, used for acute upper respiratory tract infection due to wind-heat, manifested as fever, cold feeling, sore throat, headache, stuffy nose with discharge, coughing etc.
Clear heat and toxins, relieve convulsion and rescue consciousness, used for high fever with seizures, delirium, coma due to stroke, encephalitis, meningitis, intracerebral hemorrhage, sepsis due to excessive heat inflicting the heart. 
TABLE 1 | (Continued) Summary of recommended oral CPMs in the Guidelines on Diagnosis and Management of COVID-19.

Drug name

Suhexiang Pill

\section{Compositions}

Liquidambar orientalis Mill.

Styrax tonkinensis (Pierre) Craib ex

Hartwich

Anxixiang

Bingpian

Shuiniujiao

Shexiang

Tanxiang

Chenxiang

Dingxiang

Xiangfu

Muxiang

Ruxiang

Biba

Baizhu

Hezirou

Zhusha
Borneolum Syntheticum

Cornu Bubali

Moschus

Santalum album L.

Syzygium aromaticum (L.) Merr. \&

L.M.Perry

Cyperus rotundus L.

Aquilaria sinensis (Lour.) Spreng.

Boswellia carteri Birdw.

Piper longum L.

Atractylodes macrocephala Koidz.

Terminalia chebula Retz.

Cinnabar
Aquilaria sinensis (Lour.) Spreng.

\section{Clinical indications}

Resuscitate with aromatics, facilitate flow of Qi and relieve pain, used for coma due to phlegm blocking the heart, paresis due to stroke, heat stroke, heart pain, and stomache.

TABLE 2 | Summary of recommended CPMs injection in the Guidelines on Diagnosis and Management of COVID-19.

\section{Drug name}

Compositions

\section{Clinical indications}

Clear heat and toxins, relieve coughing and diarrhea, used for bronchitis, tonsillitis, bacterial dysentery.

Xiyanping Injection Andrographolide sulfonates

Remove statisis and clear toxins, used for warm-heat conditions, manifested as fever, panting, palpitation, dysphoria due to accumulation of toxins and stasis, applicable for systemic inflammatory reaction due to infection, and for multiple organ dysfunction at the dysfunctioning stage.

Chuanxiong Conioselinum anthriscoides

'Chuanxiong'

Danshen Salvia miltiorrhiza Bunge

Danggui Angelica sinensis (Oliv.) Diels

Reduning Injection Qinghao Artemisia annua L. Jinyinhua Lonicera japonica Thunb.

Zhizi Gardenia jasminoides J.Ellis

Tanreqing Huangqin Scutellaria baicalensis Georgi

Injection Xiongdanfen Bear Bile Powder

Shanyangjiao Cornu Caprae

Jinyinhua Lonicera japonica Thunb.

Lianqiao Forsythia suspensa (Thunb.) Vahl

\begin{tabular}{|c|c|c|c|}
\hline $\begin{array}{l}\text { Xingnaojing } \\
\text { Injection }\end{array}$ & $\begin{array}{l}\text { Shexiang } \\
\text { Yujin } \\
\text { Bingpian } \\
\text { Zhizi }\end{array}$ & $\begin{array}{l}\text { Moschus } \\
\text { Curcuma aromatica Salisb. } \\
\text { Borneolum Syntheticum } \\
\text { Gardenia jasminoides J.Ellis }\end{array}$ & $\begin{array}{l}\text { Clear heat and toxins, cooling blood and remove stasis, restore consciousness, used for } \\
\text { dysfunction of Qi-blood in meridians, stasis of the brain meridian leading to stroke and coma, } \\
\text { paresis and dysarthria; headache and coma due to trauma; alcohol intoxication, headache, } \\
\text { nausea, coma with seizures, brain embolism, acute stage of intracerebral hemorrhage, head } \\
\text { trauma, acute toxicity of alcohol. }\end{array}$ \\
\hline
\end{tabular}

Shenfu Injection Hongshen Panax ginseng C.A.Mey.

Fupian Aconitum carmichaeli Debx.

Rescue Yang and Qi, tonify Qi and prevent collapse. Used for shock due to infections, loss of blood, dehydration, and for Yang (Qi) deficiency, manifested as panic, severe palpitation, coughing and panting, stomachache, diarrhea, and paresis.

Shenmai Injection Hongshen Panax ginseng C.A.Mey.

Maidong Ophiopogon japonicus (Thunb.) Ker

Gawl.

Tonify Qi and prevent collapse, increase Yin, Jin, and Mai. Used for shock due to Qi and Yin deficiency, coronary heart disease, viral myocarditis, chronic pulmonary heart disease, granulocytopenia, increases immunity of cancer patients during chemotherapy, reduce side effects of chemotherapy drugs.

\begin{tabular}{|c|c|c|}
\hline Shengmai & Hongshen & Panax ginseng C.A.Mey. \\
\hline Injection & Maidong & $\begin{array}{l}\text { Ophiopogon japonicus (Thunb.) Ker } \\
\text { Gawl. }\end{array}$ \\
\hline
\end{tabular}

Wuweizi Schisandra chinensis (Turcz.) Baill.
Tonify Qi and prevent collapse, increase Yin, used for palpitation, panting, cold limbs, perspiration, loss of pulse or myocardial infarction, cardiogenic shock, infectious shock due to Qi and Yin deficiency. 


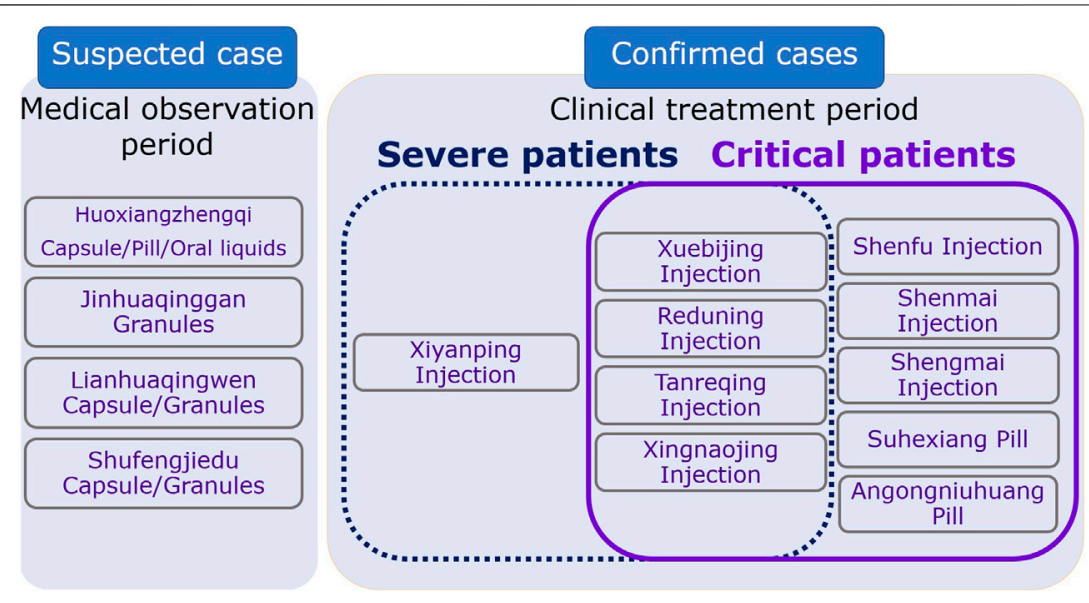

FIGURE 1 | Recommended CPMs and corresponding applicable patients with COVID-19.

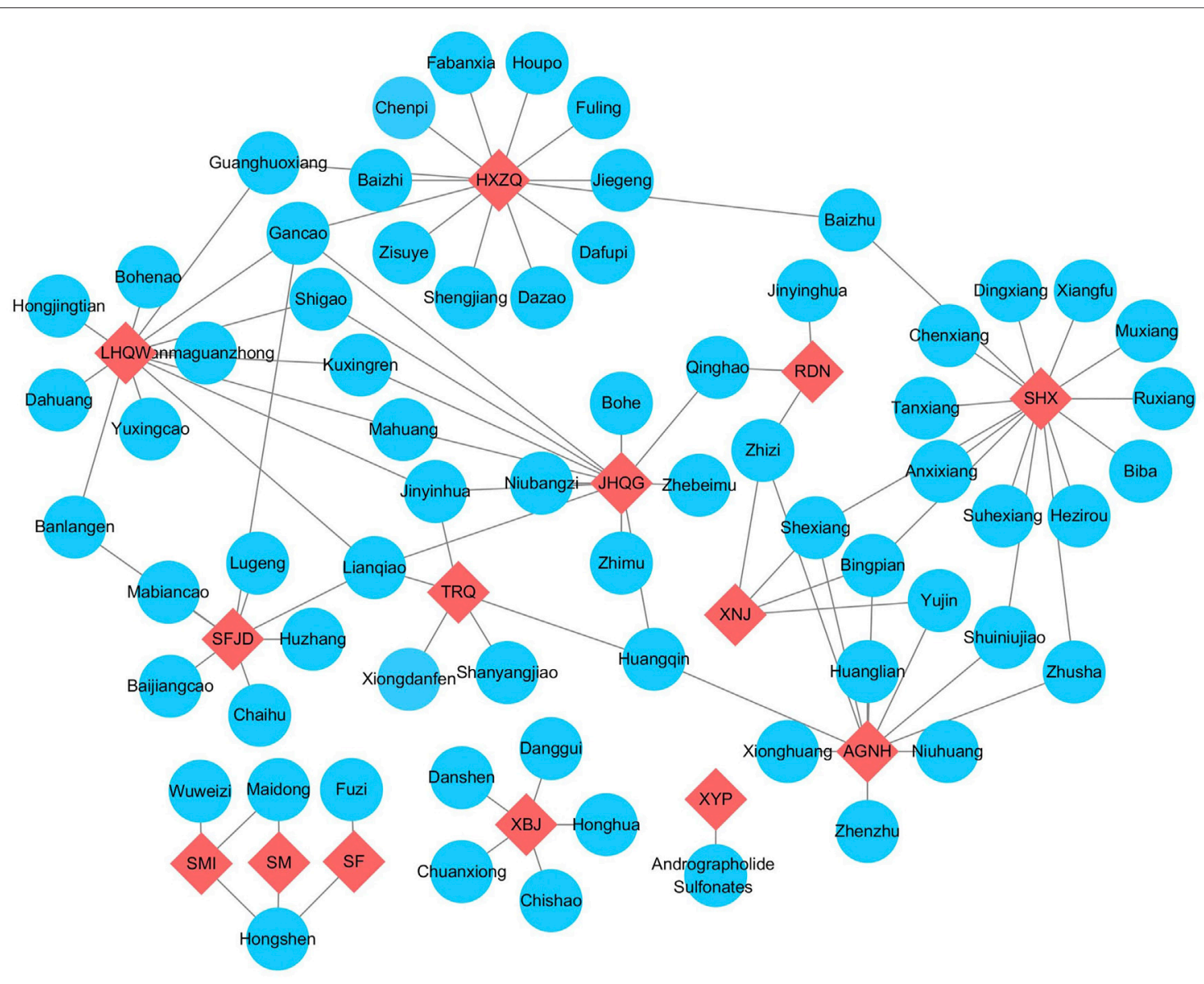

FIGURE 2 | The relationship of recommended CPMs and compositions. Note: The red nodes represented different CPMs, and compositions were labelling blue. AGNH, angong Niuhuang Pill; HXZQ, huoxiangzhengqi capsule; JHQG, jinhuaqinggan granules; LHQW, lianhuaqingwen capsule; RDN, reduning injection; SF, shenfu injection; SFJD, shufengjiedu capsule; SHX, suhexiang pill; SM, shenmai injection; SMI, shengmai injection; TRQ, tanreqing injection; XBJ, xuebijing injection; XNJ, xingnaojing injection; XYP, xiyanping injection. 
TABLE 3 | Clinical trials on CPMs for COVID-19.

\begin{tabular}{|c|c|c|c|c|}
\hline Drug name & $\begin{array}{l}\text { Registration } \\
\text { number }\end{array}$ & Public title & Study type & Study design \\
\hline $\begin{array}{l}\text { Huoxiangzhengqi oral } \\
\text { liquids }\end{array}$ & ChiCTR2000029479 & $\begin{array}{l}\text { Research for Traditional Chinese Medicine Technology Prevention and } \\
\text { Control of Novel Coronavirus Pneumonia (COVID-19) in the Community } \\
\text { Population }\end{array}$ & $\begin{array}{l}\text { Interventional } \\
\text { study }\end{array}$ & Parallel \\
\hline \multirow[t]{3}{*}{$\begin{array}{l}\text { Lianhuaqingwen Capsule/ } \\
\text { Granule }\end{array}$} & ChiCTR2000035046 & $\begin{array}{l}\text { Efficacy and outcomes of Lian-Hua Qing-Wen capsule in the treatment of } \\
\text { novel coronavirus pneumonia (COVID-19): a medical records based } \\
\text { retrospective study }\end{array}$ & $\begin{array}{l}\text { Observational } \\
\text { study }\end{array}$ & $\begin{array}{l}\text { Non randomized } \\
\text { control }\end{array}$ \\
\hline & ChiCTR2000029433 & $\begin{array}{l}\text { A randomized, open-label, blank-controlled trial for Lian-Hua Qing-Wen } \\
\text { Capsule/Granule in the treatment of suspected novel coronavirus } \\
\text { pneumonia (COVID-19) }\end{array}$ & $\begin{array}{l}\text { Interventional } \\
\text { study }\end{array}$ & Parallel \\
\hline & ChiCTR2000029434 & $\begin{array}{l}\text { A randomized, open-label, blank-controlled trial for Lian-Hua Qing-Wen } \\
\text { Capsule/Granule in the treatment of novel coronavirus pneumonia } \\
\text { (COVID-19) }\end{array}$ & $\begin{array}{l}\text { Interventional } \\
\text { study }\end{array}$ & Parallel \\
\hline Jinhuaqinggan Granules & ChiCTR2000036871 & $\begin{array}{l}\text { Multi-center, Randomized, Double Blind and Placebo Controlled Clinical } \\
\text { Trial on the Efficacy and Safety of Jinhuaqinggan Granules (JHQG) for the } \\
\text { Treatment of COVID-19 Patients. }\end{array}$ & $\begin{array}{l}\text { Interventional } \\
\text { study }\end{array}$ & Parallel \\
\hline \multirow[t]{4}{*}{ Xiyanping Injection } & ChiCTR2000030218 & $\begin{array}{l}\text { Study of pinavir/ ritonavir Tablets (Trade Name: Kelizhi) Combined with } \\
\text { Xiyanping Injection for Novel Coronavirus Pneumonia (COVID-19) }\end{array}$ & $\begin{array}{l}\text { Interventional } \\
\text { study }\end{array}$ & Parallel \\
\hline & ChiCTR2000032412 & $\begin{array}{l}\text { A medical records based retrospective study for the effectiveness and } \\
\text { safety of Xi-Yan-Ping injection combined with conventional protocol in the } \\
\text { treatment of common type novel coronavirus pneumonia (COVID-19) }\end{array}$ & $\begin{array}{l}\text { Observational } \\
\text { study }\end{array}$ & $\begin{array}{l}\text { Non randomized } \\
\text { control }\end{array}$ \\
\hline & ChiCTR2000030117 & $\begin{array}{l}\text { A multicenter, randomized, open, parallel controlled trial for the evaluation } \\
\text { of the effectiveness and safety of Xiyanping injection in the treatment of } \\
\text { common type novel coronavirus pneumonia (COVID-19) }\end{array}$ & $\begin{array}{l}\text { Interventional } \\
\text { study }\end{array}$ & Parallel \\
\hline & ChiCTR2000029756 & $\begin{array}{l}\text { Clinical study of nebulized Xiyanping injection in the treatment of novel } \\
\text { coronavirus pneumonia (COVID-19) }\end{array}$ & $\begin{array}{l}\text { Interventional } \\
\text { study }\end{array}$ & Parallel \\
\hline Xuebijing injection & ChiCTR2000030388 & $\begin{array}{l}\text { Efficacy and safety of Xue-Bi-Jing injection in the treatment of severe cases } \\
\text { of novel coronavirus pneumonia (COVID-19) }\end{array}$ & $\begin{array}{l}\text { Interventional } \\
\text { study }\end{array}$ & Parallel \\
\hline Reduning injection & ChiCTR2000029589 & $\begin{array}{l}\text { A prospective, randomized, open, multi-center clinical trial evaluating the } \\
\text { efficiency and safety of Reduning injection in the treatment of novel } \\
\text { coronavirus pneumonia (COVID-19) }\end{array}$ & $\begin{array}{l}\text { Interventional } \\
\text { study }\end{array}$ & $\begin{array}{l}\text { Non randomized } \\
\text { control }\end{array}$ \\
\hline Tanreqing Injection & ChiCTR2000029432 & $\begin{array}{l}\text { A Real World Study For the Efficacy and Safety of Large Dose Tanreqing } \\
\text { Injection in the Treatment of Patients with Novel Coronavirus Pneumonia } \\
\text { (COVID-19) }\end{array}$ & $\begin{array}{l}\text { Interventional } \\
\text { study }\end{array}$ & Sequential \\
\hline Tanreqing Capsules & ChiCTR2000029813 & $\begin{array}{l}\text { Clinical Trial for Tanreqing Capsules in the Treatment of Novel Coronavirus } \\
\text { Pneumonia (COVID-19) }\end{array}$ & $\begin{array}{l}\text { Interventional } \\
\text { study }\end{array}$ & Parallel \\
\hline Shengfu injection & ChiCTR2000030043 & $\begin{array}{l}\text { Shen-Fu injection in the treatment of severe novel coronavirus pneumonia } \\
\text { (COVID-19): a multicenter, randomized, open-label, controlled trial }\end{array}$ & $\begin{array}{l}\text { Interventional } \\
\text { study }\end{array}$ & Parallel \\
\hline
\end{tabular}

product bind to the main protease (Mpro) and angiotensin converting enzyme II (ACE2) of SARS-CoV-2 with high affinity, through which COVID-19 is inhibited (Ling et al., 2020; Xia et al., 2020). A pharmacodynamic study showed that Lianhuaqingwen granules significantly inhibited the replication of COVID-19 and transformed the morphology of this virus in vitro ( $\mathrm{Li}$ et al., 2020b). Another study showed that Lianhuaqingwen capsules inhibited the replication of multiple influenza viruses in vitro and the $50 \%$ inhibitory concentration (IC50) of Lianhuaqingwen capsules was in the range of $0.35 \mathrm{mg} /$ $\mathrm{ml} 2 \mathrm{mg} / \mathrm{ml}$ (Ding et al., 2017). A prospective, multi-centered, randomized controlled clinical study investigated the recovery rate of main symptoms including fever, fatigue, and coughing by dividing 284 COVID-19 patients from 23 hospitals of nine provinces in China into the conventional treatment group and the Lianhuaqingwen granule group. It was found that the Lianhuaqingwen group had a cure rate of $91.5 \%$, which was significantly higher than that of the conventional group- $82.4 \%$ (independent $t$ test, $p=0.022$ ). The median time of recovery was significantly shorter in the Lianhuaqingwen group than in the conventional group ( 7 vs 10 days, $p<0.001$ ). This was also observed in the duration of fever ( 2 vs 3 days, $p<0.001)$, fatigue ( 3 vs 6 days, $p<0.001$ ), and coughing ( 7 vs 10 days, $p<0.001$ ). $83.8 \%$ of patients in the Lianhuaqingwen group showed improvement on chest computed tomography compared to $64.1 \%$ in the conventional group ( $p<0.001$ ), and $78.9 \%$ of patients were cured in the Lianhuaqingwen group compared to $66.2 \%$ in the conventional group ( $p=0.017$ ). However, no difference was found in the proportion of patients progressed into the critical stage or in the viral test $(p>0.05)$ (Hu et al., 2020). 
Another study examined 151 severe patients with confirmed COVID-19 on their coagulation, white cell count, and prognosis after 25 days of treatment with ribavirin, ritonavir, umifenovel, and Lianhuaqingwen. It was found that coagulation and white cell count were significantly improved after the treatment and their prognosis was good. Therefore, it was recommended to be the first line treatment for this type of patients (Li, 2020). In a study on suspicious COVID-19 patients, the proportions of patients without fever, coughing, and malaise were 86.7, 55.6, and $82.5 \%$ in the Lianhuaqingwen group with conventional treatment and Lianhuaqingwen after receiving the treatment compared with $67.7,30.6$, and $58.6 \%$ in the conventional treatment group (all $p<0.05$ ). Similar results were found in the disappearance of shortness of breath and moist rales between the two groups ( 68.2 vs $20.0 \%, 56.0$ vs $20.0 \%$, $p<$ 0.05). No significant difference was found in the duration of fever and ratios of patients progressed to the critical stage ( $\mathrm{Lv}$ et al., 2020). In a retrospective study on $214 \mathrm{mild} /$ general COVID-19 patients who were treated with arbidol or in combination with Lianhuaqingwen capsules, it was found that patients treated with arbidol and symptomatic therapy had a higher incidence of progressing to the critical stage than those treated with both arbidol, symptomatic therapy, and Lianhuaqingwen $\left(\chi^{2}=16.823\right.$, $p=0.001)$. There was no significant difference in the duration of admission $\left(\chi^{2}=2.889, p=0.409\right)$ (Yu et al., 2020). Another study reported positive results on fever, coughing, malaise, and other symptoms after treatment with Lianhuaqingwen, but there was no control group in that study and the level of evidence is low (Cheng and Li, 2020).

Huoxiangzhengqi pills were first recorded in $<$ Taipinghuiminhejijufang (Formularies of the Bureau of People's Welfare Pharmacies) $>$. This herbal recipe is known for resolving dampness with aromatics and dispersing cold as well as regulating the stomach. Modern pharmacological research has shown that this herbal product has anti-spasm, analgesic, anti-bacterial, anti-viral, anti-emetic, and regulating functions of the stomach and the intestines (Huo et al., 2020). Network pharmacology and molecule docking techniques revealed that compounds in Huoxiangzhengqi oral liquid took its anti-viral effect by modulating multiple pathways, such as prostaglandinendoperoxide synthase (PTGS-2), Heat Shock Protein 90 Alpha Family Class B Member 1 (HSP90AB1), and calmodulinregulated spectrin-associated protein 2 (CAMSAP2), after binding to ACE2 (Deng et al., 2020). In a prospective, randomized, parallel controlled preventive study, Huoxiangzhengqi oral liquid was prescribed in combination with Jingaojiere granules to COVID-19 patients. A total of 22,065 recipients were divided into two groups: the health briefing group and the herbal group. The latter group was also briefed with hygiene measures. During the 14 days follow up period, neither of the groups had COVID-19 patients, but the herbal group had fewer participants suffering from the cold than the health briefing group $(p<0.05)$. Herbal products protected $91.8 \%$ of the population, especially people aged $16-60$ years (Yan et al., 2020). The other study showed that Huoxiangzhengqi pills along with Lianhuaqingwen granules decreased the use of macrolides by COVID-19 patients, but did not show superiority in disease deterioration than conventional treatment. However, combined herbal therapy did decrease the proportion of patients progressed to the critical stage, demonstrating the potential of combined use of herbal products and conventional therapies in improving the outcomes of COVID-19 patients (Xiao et al., 2020).

Jinhuaqinggan granules derived from Yinqiaosan and Maxingshigantang by removing some herbs and adding others. This herbal product is known for dispelling the wind, ventilating the lungs, clearing the heat and toxins (Mao et al., 2020). Network pharmacology research found that it had multiple effective compounds, like formononetin, stigmasterol, $\beta$-quebrachol, icaritin, etc., which regulated multiple pathways, such as PTGS2, HSP90AB1, HSP90AA1, PTGS1, through binding to SARS-CoV-2 3C-like protease (3CL ${ }^{\text {pro }}$ ) and $\mathrm{ACE} 2$, resulting in the control of COVID-19 (Gong et al., 2020). In another study, 123 COVID-19 patients were randomly allocated to the combined treatment group (Jinhuaqinggan granules and conventional treatment) and the conventional treatment group in a ratio of 2:1. After 5 days treatment, the combined treatment group showed significant improvement in fever (80.3 vs $53.1 \%$, $p<0.05)$, coughing ( 66.1 vs $42.9 \%, p<0.05)$, malaise $(77.6$ vs $53.8 \%, p<0.05$ ), expectoration ( 85.3 vs $46.2 \%, p<0.05$ ). Scores in the TCM syndrome scale and Hamilton depression scale were significantly lower in the combined treatment group than in the conventional group $(p<0.01)$ (Duan et al., 2020). In a retrospective study, 80 COVID-19 patients were divided into two groups, receiving Jinhuaqinggan granules and conventional treatment, respectively. It was found that the duration of positive virus detection was shorter in the Jinhuaqinggan group than in the conventional group $(7 \pm 4$ days vs $10 \pm 4$ days, $p<0.05)$. The virus clearance rate was higher ( 56.82 vs $27.78 \%, p<0.05)$ and the time to recover as shown on chest computed tomography (CT) was shorter $(8 \pm 4$ days vs $10 \pm 5$ days, $p=0.021)$ in the Jinhuaqinggan group than in the conventional group, respectively (Liu et al., 2020).

Shufengjiedu capsules are one of the essential herbal recipes in managing acute upper respiratory tract infection. Animal experiments, network pharmacology, as well as genetic studies showed that this herbal product had multi-component, multitarget, and multi-pathway characters (Zhang et al., 2016). In the acute lung injury model, Shufengjiedu capsules were able to suppress inflammation induced by LPS (lipopolysaccharide) and to relieve lung injuries due to endotoxins, which might be mediated by inhibition of the mitogen-activated protein kinases $(\mathrm{MAPK}) /$ nuclear factor kappa B (NF- $\mathrm{B}$ ) signaling pathway and downregulation of NF- $\kappa \mathrm{B}$ mRNA (Tao et al., 2014). Network pharmacology and molecule docking techniques revealed that compounds, extracted from this herbal product including quercetin, luteolin, kaempferol, wogonin and acacetin, were able to regulate multiple pathways through binding to key target proteins like interleukin 6 (IL-6), albumin (ALB), MAPK3, and others, resulting in the control of COVID-19 (Shen et al., 2020). In a retrospective study on 200 general COVID-19 patients who were treated with arbidol hydrochloride or in combination with Shufengjiedu capsules, it was found that patients treated with arbidol hydrochloride and 
Shufengjiedu capsules had a shorter time in defervescence, larger white blood cell counts, higher percentages of lymphocytes and lower serum C-reactive protein (CRP) as well as IL-6 than those treated with arbidol hydrochloride alone, respectively. As expected, the combined treatment group had a higher percentage of clear chest CT than the arbidol hydrochloride group (Chen et al., 2020a).

\section{HERBAL PRODUCTS FOR SEVERE OR CRITICAL COVID-19 PATIENTS}

Xiyanping injection is a single compound formula obtained after sulfonating Andrographolide extracted from Andrographis paniculate. It has non-specific, anti-inflammatory, anti-viral effect and has been recommended to treat $\mathrm{H} 1 \mathrm{~N} 1$ influenza, epidemic colds (influenza B), H7N9 avian influenza, community acquired pneumonia (Wang ZF. et al., 2019). To investigate the anti-inflammatory effect of Andrographolide, mRNA levels of inflammatory biomarkers, such as toll-like receptor 4 (TLR4), CD14 and myeloid differentiation factor 2 (MD2), were examined in a rat model of acute lung injury. It was found that Andrographolide decreased mRNA levels of TLR4, CD14 and MD2 in LPS induced acute lung injury (Xu et al., 2015), suppressed inflammation mediated by NF- $\kappa B$ and MAPK (Peng et al., 2016), and alleviated pathologies of the lungs. A meta-analysis study on community acquired pneumonia showed that Reduning injection, Yanhuning injection, Xiyanping injection, and Tanreqing injection, when used in combination with conventional treatment, showed better therapeutic effect than conventional treatment alone. Tanreqing plus conventional treatment showed the best result in reducing the duration of fever and the average length of stay. Xiyanping plus conventional treatment showed the best result in reducing moist rales in the lungs (Li et al., 2017). In a prospective, randomized controlled study on hand-foot-and-mouth disease, 451 severe pediatric patients were divided into the Xiyanping or Reduning plus conventional treatment group and the conventional treatment group. It was found that the conventional treatment group had a longer half time of defervescence ( 40.4 vs $27.2 \mathrm{~h}, p<0.01$ ), a lower clearance of rashes (43.6 vs $29.5 \%, p<0.01$ ), higher proportions of patients without coughing, malaise, fragility, agitation or irritability (32.6 vs $19.2 \%, p<0.01)$ than the combined group, respectively. Xiyanping had similar therapeutic effect on mild hand-foot and mouth disease (Zhang G. et al., 2017). A meta-analysis of the therapeutic effect of Xiyanping plus azithromycin on Chinese pediatric pneumonitis due to mycoplasm infection included nine trials and 963 patients. It showed that Xiyanping plus azithromycin were superior in duration of admission, time to defervescence, time to stop coughing, time to disappearance of moist rales than azithromycin alone (Li Q. et al., 2019).

Xuebijing injection is comprised of extracts from Carthamus tinctorius L. (Honghua), Paeonia lactiflora Pall. (Chishao), Conioselinum anthriscoides "Chuanxiong" (Chuanxiong), Salvia miltiorrhiza Bunge (Danshen), Angelica sinensis (Oliv.) Diels (Danggui). It promotes blood circulation to remove blood stasis and clears toxins, for which it has been used to treat systemic inflammatory response syndrome and multiple organ dysfunction at the dysfunctioning stage (Shi et al., 2020). Its therapeutic effect was recognized by clinical experts (Li C. et al., 2018; Song et al., 2018). Network pharmacology research showed that multiple compounds shared a number of targets with COVID-19. Among them, IL6, tumor necrosis factor alpha (TNFa), MAPK1, MAPK14, MAPK8, PTGS2, IL2 and PPARG were the predominant targets for Xuebijing to treat COVID-19. The major effective compounds of this herbal product, including luteolin, quercetin, kaempferol, $\beta$-sitosterol, stigmasterol, were able to bind to 2019-nCoV 3CLpro and ACE2 to resist COVID19 (Huang et al., 2020). In a study on Xuebijing injection, 60 severe COVID-19 patients were divided into conventional treatment group, $50 \mathrm{ml}$ Xuebijing group, and $100 \mathrm{ml}$ Xuebijing group on a 1:1:1 basis. After 8 days treatment, the Acute physiology and chronic health evaluation II (APACHE-II) score was lower in the $100 \mathrm{ml}$ Xuebijing group than in the conventional treatment group and the $50 \mathrm{ml}$ Xuebijing group [APACHE-II score: $12.3 \pm 1.5$ (100 ml Xuebijing group) vs $16.5 \pm$ 1.6 (conventional treatment group) vs $15.9 \pm 1.4(50 \mathrm{ml}$ Xuebijing group), $p<0.05$, respectively). After treatment, the COVID-19 nucleic acids of some patients turned negative, but there was no significant difference between groups. The $100 \mathrm{ml}$ Xuebijing group showed significantly greater improvement than the $50 \mathrm{ml}$ Xuebijing group $(p<0.05)$ and the conventional treatment group $(p<0.05)$ (Wen et al., 2020). In another study, 42 COVID-19 patients received Xuebijing plus conventional treatment (including electrolytes, blood glucose and blood pressure management, nutritional support, oxygen therapy and antiviral treatment) and patients in the control group received conventional treatment alone. After 7 days treatment, the combined group showed significant improvement in IL-6 and temperature than the conventional group with a larger reduction in temperature in the combined group $(p<0.05)$, especially in severe patients. The proportion of patients with improvement in chest CT and COVID-19 nucleic acids turning negative was higher in the combined group than in the conventional group, but this difference was not statistically significant $(p>0.05)$ (Guo et al., 2020). In another study, 60 patients with COVID-19 were divided into the conventional treatment group and the Xuebijing group (conventional treatment plus Xuebijing). It was found that Xuebijing significantly suppressed IL-6, IL-8 and TNF- $\alpha$ ( $p<$ $0.05)$. There was no significant difference in adverse events such as allergic shock (Luo et al., 2021). In a prospective, randomized controlled study on 710 patients with community acquired pneumonitis, it was shown that Xuebijing significantly decreased the mortality rate on day 28 (15.87 vs $24.63 \%, p=$ 0.006 ), the time in need of mechanical ventilation (11 vs 16.5 days, $p=0.012$ ), and duration of admission (12 vs 16 days, $p=0.004$ ) than placebo, respectively. There was no significant difference in adverse events between the two groups (Jiang et al., 2019; Song et al., 2019).

Reduning injection is comprised of extracts from Artemisia annua L. (Qinghao), Lonicera japonica Thunb. (Jinyinhua), Gardenia jasminoides J.Ellis (Zhizi). It has been widely used for upper respiratory tract infection due to its superiority in 
clearing the heat, dispelling the wind, and clearing toxins. Its therapeutic effect on COVID-19 might be attributed to its antiinflammatory and multi-target, multi-pathway immune modulating characters (Jiang et al., 2019). Interacting proteins of SARS-CoV-2 and humans have been mapped, among which human proteins like ATP binding cassette C1 (ABCC1), sigma receptor 1 (SigmaR1), and inosine monophosphate dehydrogenase type II (IMPDH2) were targeted by Reduning to interrupt interactions between viral and human proteins. As a result, the invasion process of COVID-19 was discontinued. As a result, infection by COVID-19 was prevented (Chen S. J. et al., 2020). In a multi-centred, randomized clinical trial, the therapeutic effect and safety of Reduning were investigated in 12 hospitals in China. Patients receiving Reduning injections and conventional treatment had a higher percentage of mitigated symptoms after 14 days treatment compared with those receiving conventional treatment alone [full-analysis set (FAS): 84.4 vs $60.0 \%, p=0.0004]$. They also had shorter median time to symptom alleviation ( $143 \mathrm{~h}$ vs $313.5 \mathrm{~h}, p<0.001$ ), time to nucleic acid turning negative ( $146.5 \mathrm{~h} v s 255.5 \mathrm{~h}, p<0.001$ ), length of stay ( $4.1 \mathrm{~d} v s 18.1 \mathrm{~d}, p<0.001$ ), and time to defervescence $(29 \mathrm{~h} v s 71 \mathrm{~h}, p<0.001)$ than the control group. No significant difference in adverse events were found between these two groups (3.9\% vs 8.8\%, $p=0.383$ ) (Xu et al., 2021). In a previous multi-centred, stratified randomized, double blind, parallel controlled study on hand-foot and mouse disease, patients were randomly divided into three groups with 120 in each group. The first group received conventional treatment, the second group received Reduning injection, and the third group received Reduning plus conventional treatment. It was found that the combined group was more superior in defervescence than the conventional group, and more superior in managing rashes than the conventional group and the Reduning group. But there was no significant difference in managing stomatological ulcer between these three groups (Zhang et al., 2013). A metaanalysis on Reduning managing acute tracheobronchitis showed that Reduning increased the effectiveness rate, decreased the duration of fever, coughing, rales in the lungs, and the incidence of adverse events which were minor in nature. However, the quality of this study was low, and its conclusion should be verified in larger-sampled, well designed, standardized clinical trials (Dang et al., 2019).

Tanreqing injection is the modified form of Shuanghuanglian by adding two heat clearing herbs-cornu gorais and bear gall powder. It clears heat, resolves phlegm, and clears toxins, for which it has been used to treat wind-warm lung heat disease (main symptoms include fever, coughing, chest pain) and lung diseases due to phlegm and heat blocking. The major compounds include baicalin, wogonoside, wogonin, ursodeoxycholic acid, chenodeoxycholic acid, chlorogenic acid, neochlorogenic acid, cryptochlorogenic acid, forsythiaside and others (Li C. et al., 2019). The therapeutic mechanism might be related to the high affinity of its major effective compounds like kaempferol, quercetin, baicalein, luteolin, and wogonin with SARS-CoV-2 $3 \mathrm{CL}^{\text {pro }}$ (Kong et al., 2020). Previous studies showed that Tanreqing has been prescribed with antibiotics to increase the bactericidal effect and to decrease the resistance of bacteria to antibiotics (Chang, 2017; Tang et al., 2019). When used with vancomycin or linezolid at concentrations lower than their minimal inhibitory concentrations for methicillin-resistant Staphylococcus aureus in vitro, the synergistic bactericidal effect was stronger than each antibiotic when used alone (Yang et al., 2018). There is only one study which has reported the therapeutic effect of Tanreqing capsules on COVID-19. In this retrospective cohort study on 82 mild and moderate COVID-19 patients, it was found that the time to negative COVID-19 DNA in the stool (4 days vs 9 days, $p=$ 0.047 ) and the duration of throat-stool negative COVID-19 DNA ( 0 vs. 2 days, $p=0.042$ ) were shorter in the Tanreqing group than in the control group. These might be related to the increase in $\mathrm{CD}^{+} \mathrm{T}$ cells (Zhang et al., 2020a). In a meta-analysis on acute onset of chronic bronchitis, 23 randomized controlled trials (RCTs) were included with 1,901 participants. It was found that time to defervescence was significantly shorter in the Tanreqing plus conventional treatment group than in the conventional treatment group. Similar superiority was also observed in the total effective rate. When used along with levofloxacin, cefuroxime, cefperazone- sulbactam, Tanreqing showed synergy with these antibiotics. Though this superiority was observed in this meta-analysis, the conclusion should be verified in future large-scaled, high-quality studies due to the small sample sizes of included studies and the low quality of study design (Gao et al., 2019).

Xingnaojing injection is derived from the classical herbal decoction Angongniuhuangwan after refining the major components of this recipe using modern pharmacological techniques. It clears the heat and toxins, cools and invigorates the blood, and revitalize the brain. It has been widely used for intracerebral hemorrhage, brain ischemia, and other neurological diseases (Zhang S. et al., 2017). In vitro experiments showed that the therapeutic effect on loss of consciousness is due to its antineurotoxicity induced by excitatory amino acids, radical scavenging, cytokine suppressing, and apoptosis related gene regulating characters ( $\mathrm{Ma}$ et al., 2018). It can be used for COVID-19 patients with high fever and disturbance in consciousness, but there is no such report. In a meta-analysis on Xingnaojing managing coma due to high fever, stroke, and intoxication, it was found that Xingnaojing was superior in improving consciousness for such patients (Wu et al., 2016). An ongoing clinical trial- Xingnaojing for moderate-to-severe acute ischemic stroke (Lai et al.) aimed to investigate whether Xingnaojing is able to improve outcomes of ischemic stroke patients at 3 months after stroke onset by running a multicentred, prospective, randomized controlled, open label trial. The result is still pending (Lai et al., 2017).

Angongniuhuang pills and Suhexiang pills can be used for resuscitation of severe or critical COVID-19 patients. Both of them are of rapid effect and potent in revitalizing the brain and are used for resuscitation of patients due to diverse critical conditions (Bian and Zhang, 2016; Liang et al., 2019). Based on the fact that the majority of patients with critical conditions have loss of consciousness and accompanying dysphagia, there is an increased risk of aspiration and choking when taking oral dosage forms. Therefore, these herbal products can be dissolved 
in water and dosed through the naso-gastric tube to the stomach of patients. Both of them are used for critical patients with internal closure and external detachment. However, Angongniuhuang pills are for internal closure with heat, and Suhexiang pills for internal closure with cold (Wang et al., 2020). Currently, there is no report on their therapeutic effect on severe COVID-19. In a meta-analysis on Angongniuhuang pills for stroke, 18 clinical trials including 1,601 participants were studied. It was found that Angongniuhuang pills significantly improved outcomes of ischemic stroke patients [relative risk (RR): 1.27; 95\% confidence interval (Health Emergency Committee of The Chinese Research Hospital Association, 2019): 1.14-1.41] and ICH patients (RR: 1.26; 95\%CI: 1.14-1.38), decreased the neurological deficit scores of ischemic stroke patients [weighted mean difference (WMD) -3.52 ; $95 \% \mathrm{CI}:-5.51$ to -1.54 ] and $\mathrm{ICH}$ patients (WMD -3.64 ; $95 \%$ CI: -4.97 to -2.31 ), and improved the Glasgow Coma scores of such patients (WMD 1.18; 95\% CI: 0.79-1.56; WMD 2.28; 95\% CI: 1.37-3.19) (Liu et al., 2019).

In addition, another three injections containing ginseng have been used for patients with collapse syndrome. They are Shenfu injection, Shenmai injection, and Shengmai injection (Li et al., 2016; Cao et al., 2019). Shengmai injection is derived from Shengmaisan and Shenmai injection from Maidongyin, the latter has one herb-Schisandra chinensis (Turcz.) Baill. (Wuweizi) less than the former one. Due to the similarity to each other, they are of similar effect on certain diseases. Deficiency in Yang or Yin should be differentiated before determining which to prescribe. Patients with Yang deficiency can be prescribed Shenfu injection, those with Yin deficiency Shengmai or Shenmai injection. In a meta-analysis including 30 RCTs and 2,038 participants, it was found that Shenmai injection plus conventional treatment was more superior in managing patients with shock (septic shock, cardiogenic shock, hypovolemic shock, neurogenic shock, allergic shock) than conventional treatment ( $\mathrm{Li}$ X. et al., 2018). In another metaanalysis including 23 RCTs and 1,804 participants, Shengmai injection plus conventional treatment significantly improved pulmonary function, blood-gas index, immunoglobins, and time to disappearance of rales of patients with chronic obstructive pulmonary diseases (COPD). It also significantly decreased the COPD Assessment Test (CAT) score, modified Medical Research Council (mMRC), and the average duration of admission. Currently, little evidence is available for their use on COVID-19 (Huang et al., 2019). Further pharmacological research and clinical trials are needed to confirm their efficacy in managing COVID-19.

\section{ADVERSE EVENTS AND OTHER NOTES}

CPMs are made of natural herbs processed using traditional techniques. Their clinical efficacy and adverse events are not well known, which misleads the public to believe that herbs are safe and non-toxic. As adverse events of drugs are closely surveilled, more and more studies are available to report the potential toxicity and adverse events of herbs. Compared with other forms of herbal products, injections are more likely to result in adverse events, especially severe adverse events, such as allergic shock. Previous studies have shown that over $70 \%$ of adverse events of Xuebijing (Guo et al., 2017), Reduning (Sun et al., 2019), Shengmai (Cheng, 2011), Shenmai (Xiang et al., 2017), Xiyanping (Chen et al., 2018) occurred in the first half an hour. A large-scale, multi-centred Chinese study reported the incidences of adverse events and adverse reactions of Tanreqing, which were 1.4 and $0.3 \%$, respectively. The most common adverse reaction was changes in the skin or the subcutaneous tissue. The majority $(72.8 \%)$ of these reactions occurred in the first 2 hours after Tanreqing injection. Among these patients, two thirds of them can be treated with externally used drugs. Increased adverse reactions were related to history of food allergy [odds ratio (Lai et al.) 4.50, 95\% CI: 1.35-15.00], drug allergy (OR 2.77, 95\% CI: 1.56-4.94), and rapid infusion of these products (OR 2.10, 95\% CI: 1.27-3.50) (Li XX. et al., 2019). A meta-analysis on Xuebijing showed that it has an incidence of adverse reactions of $5.62 \%$, allergy of $3.16 \%$, and the incidence of allergy increased as dosages and age increased (Wang C. et al., 2019). A meta-analysis study showed that Xiyanping injection, when used in combination with antibiotics to treat community acquired pneumonia, elicited similar adverse reactions to antibiotics alone. These reactions included gastrointestinal discomfort, dizziness, rashes, and phlebitis (Wang et al., 2018).

Certain herbs have toxic compounds which may lead to adverse reactions. For example, Suhexiang pills and Angongniuhuang pills have cinnabar ( $\mathrm{HgS})$ and realgar $\left(\mathrm{As}_{2} \mathrm{~S}_{2}\right)$, which cannot be used for a long term (Zhao et al., 2015). When heated, both cinnabar and realgar showed increased toxicity. Hence, they cannot be boiled, instead they can be mixed with warm water before use (Zhang et al., 2020b). Huoxiangzhengqi oral liquid has ethanol inside, which reacts with metronidazole, furazolidone, tolbutamide, and some cephalosporins with a methiazolium side chain, leading to disulfiram-like reactions which are similar to drunken behaviors (Liu and Xie, 2017). In addition, there is a lack of similar studies on certain populations, like pregnant women, lactating women, children, elderlies, and those with liver and kidney dysfunction. Therefore, caution should be taken when using herbal products. More challenges will be encountered in the prevention of COVID-19 using TCM methods, which require pharmacologists or pharmacists to provide more precise and complete service to clinicians. This will guarantee the safe prescription and efficacy of herbal products in fighting against COVID-19. In the meantime, correct clinical diagnosis is essential in managing COVID-19 patients, which will facilitate the correct use of both herbal products and western medicines with an aim to minimize the side effects and maximize the therapeutic effect. This will reflect the importance of TCM in fighting against COVID-19.

\section{CONCLUSION}

Current clinical studies on COVID-19 treated with TCM have certain limitations, such as small numbers of trials, small sample 
sizes, low quality of methodologies, and others. Large-scaled, randomized controlled, real-world studies are still lacking, especially as adjuvant therapies for critical patients (Suhexiang pills, Angongniuhuang pills, Shenfu injection, Shengmai injection, Shenmai injection). The present study only reviewed 14 herbal products recommended by the < Guidelines for diagnosis and management of COVID-19 ( $8^{\text {th }}$ edition) $>$. In fact, there are many more herbal products that might be effective in treating COVID-19 (Su et al., 2020; Ye et al., 2020; Zhao et al., 2020). As convenient, highly effective, and modern TCM products, herbal products have shown excellent clinical efficacy and tremendous potential in COVID-19 prevention and treatment. It is likely to become an alternative in fighting against this pandemic for both suspected patients or populations at a high risk.

\section{REFERENCES}

Bian, J., and Zhang, H. Y. (2016). Study on Ancient and Modern Application of Suhexiang Pill. Clin. J. Trad Chin. Med. 28, 875-878. doi:10.16448/ j.cjtcm.2016.0310

Cao, Z., Pan, J., Li, N., Qu, X., and Han, D. (2019). Modern Pharmacological Effect and Mechanism of Shengmai San. Chin. J. Exp. Trad Med. Form 25, 212-218. doi:10.13422/j.cnki.syfjx.20192208

Chang, J. (2017). Effect of Tanreqing Injection Combined with Antibiotics on the Cure Rate and Recurrence Rate of Bacterial Pneumonia. Chin. Remed Clin. 17, 584-586. doi:10.11655/zgywylc2017.04.050

Chen, J., Lin, S., Niu, C., and Xiao, Q. (2020a). Clinical Evaluation of Shufeng Jiedu Capsules Combined with Umifenovir (Arbidol) in the Treatment of Commontype COVID-19: a Retrospective Study. Expert Rev. Respir. Med. 15, 257-265. doi:10.1080/17476348.2020.1822741

Chen, J., Wang, W. Q., Shi, C. Y., and Fang, J. G. (2020b). Thoughts on Prevention and Treatment of Coronavirus Disease 2019 (COVID-19) by Traditional Chinese Medicine. Chin. Trad Herb Drugs 51, 1106-1112. doi:10.7501/ j.issn.0253-2670.2020.05.003

Chen, S., Kwong, J. S. W., Zheng, R., Wang, Y., and Shang, H. (2018). Normative Application of Xiyanping Injection: A Systematic Review of Adverse Case Reports. Evid. Based Complement. Alternat Med. 2018, 4013912. doi:10.1155/ 2018/4013912

Chen, S. J., Cui, M. C., Dong, K. H. Z. L., and Chen, H. J. (2020). Mechanism of Antiviral( SARS-CoV-2) Action of Re Du Ning Injection Based on SARS-CoV2 Protein-Protein Interaction Map. Pharmacol. Clin. Chin. Mat Med 36, 18-23. doi:10.13412/j.cnki.zyyl.20201110.003

Cheng, D. Z., and Li, Y. (2020). Clinical Effectiveness and Case Analysis in 54 NCP Patients Treated with Lanhuaqingwen Granules. World Chin. Med. 15, 150-154. doi:10.3969/j.issn.1673-7202.2020.02.006

Cheng, M., Jiang, C., and Huang, P. (2011). 1012 Cases of Adverse Reactions/event of Shengmai Injections. Anhui Med. Pharm. J. 15, 250-253. doi:10.3969/ j.issn.1009-6469.2011.02.058

Dang, J. J., Lyu, J., Sun, M. H., and Wang, L. X. (2019). [Systematic Review and Meta-Analysis of Effect of Reduning Injection in Treating Acute TrachealBronchitis]. Zhongguo Zhong Yao Za Zhi 44, 5294-5302. doi:10.19540/ j.cnki.cjcmm.20191008.501

Deng, Y. J., Liu, B. W., He, Z. X., Liu, T., Zheng, R., Yang, A. D., et al. (2020). Study on Active Compounds from Huoxiang Zhengqi Oral Liquid for Prevention of Coronavirus Disease 2019 (COVID-19) Based on Network Pharmacology and Molecular Docking. Chin. Trad Herb Drugs 51, 1113-1121. doi:10.7501/ j.issn.0253-2670.2020.05.004

Ding, Y., Zeng, L., Li, R., Chen, Q., Zhou, B., Chen, Q., et al. (2017). The Chinese Prescription Lianhuaqingwen Capsule Exerts Anti-influenza Activity through the Inhibition of Viral Propagation and Impacts Immune Function. BMC Complement. Altern. Med. 17, 130. doi:10.1186/ s12906-017-1585-7

\section{AUTHOR CONTRIBUTIONS}

YW searched for related articles. PZ proofread the article and revised the article. All authors have read and approved the article.

\section{FUNDING}

This work was supported by a research project of Class A project of key medical disciplines in Yangpu District (YP10ZA08) Shanghai Hongkou District Municipal Health and Family Planning Commission (1802-1), Shanghai Municipal Committee of Science and Technology (grant No. 19401935700); cultivation of outstanding young talents in clinical medicine in Hongkou District (HKYQ 2018-01).

Duan, C., Xia, W., Zheng, C., Sun, G., Li, Z., and Li, Q. L. (2020). An Observational Study on Jinhuaqing Granules for COVID-19 Patients. J. Trad Chin. Med. 61, 1473-1477. doi:10.13288/j.11-2166/r.2020.17.001

Gao, L. N., Lyu, J., Wang, Z. F., Yu, D. D., and Sun, M. H. (2019). [Meta-analysis of Randomized Controlled Trials on Effect of Tanreqing Injection Combined with Western Medicine on Acute Exacerbation of Chronic Bronchitis]. Zhongguo Zhong Yao Za Zhi 44, 5313-5321. doi:10.19540/j.cnki.cjcmm.20190924.501

Gong, P. Y., Guo, Y. J., Li, X. P., Wang, N., and Gu, J. (2020). Exploring Active Compounds of Jinhua Qinggan Granules for Prevention of COVID-19 Based on Network Pharmacology and Molecular Docking. J. Chin. Med. Mat 51, 1685-1693. doi:10.7501/j.issn.0253-2670.2020.07.002

Guo, H., Zheng, J., Huang, G., Xiang, Y., Lang, C., Li, B., et al. (2020). Xuebijing Injection in the Treatment of COVID-19: a Retrospective Case-Control Study. Ann. Palliat. Med. 9, 3235-3248. doi:10.21037/apm-20-1478

Guo, H., Ren, Y., Wang, P., Fu, Q., and Shao, Y. (2017). Literature Review on Adverse Reactions Induced by Xuebijing Injection. Anhui Med. Pharm. J. 21, 1548-1551. doi:10.3969/j.issn.1009-6469.2017.08.053

Health Emergency Committee of The Chinese Research Hospital Association (2019). Expert Consensus on the Clinical Application of Xingnaojing Injection in the Treatment of Acute and Serious Diseases. Chin. J. Hyg. Resc (Electronic Edition) 5, 65-70. doi:10.3877/cma.j.issn.2095-9133.2019.02.001

Hu, K., Guan, W. J., Bi, Y., Zhang, W., Li, L., Zhang, B., et al. (2020). Efficacy and Safety of Lianhuaqingwen Capsules, a Repurposed Chinese Herb, in Patients with Coronavirus Disease 2019: A Multicenter, Prospective, Randomized Controlled Trial. Phytomedicine, 153242. doi:10.1016/j.phymed.2020.153242

Huang, A. H., Zhang, Y., Li, X. F., Cui, L. Y., Li, W., and Liu, J. B. (2020). Preliminary Exploration of the Mechanism of Xuebijing in the Treatment of Novel Coronavirus Pneumonia Based on Network Pharmacology and Molecular Docking Technology. J. Chin. Med. Mat 43, 2325-2332. doi:10.13863/j.issn1001-4454.2020.09.047

Huang, X., Duan, X., Wang, K., Wu, J., and Zhang, X. (2019). Shengmai Injection as an Adjunctive Therapy for the Treatment of Chronic Obstructive Pulmonary Disease: A Systematic Review and Meta-Analysis. Complement. Ther. Med. 43, 140-147. doi:10.1016/j.ctim.2019.01.020

Huo, Z. P., Liu, Y. X., Hao, L., Wang, Y., He, Y., Zhou, S. P., et al. (2020). Research Progress on Potential Application of Huoxiang Zhengqi in Prevention and Treatment of Coronavirus Disease 2019. Drugs \& Clinic 35, 405-410. doi:10.7501/j.issn.1674-5515.2020.03.003

Jiang, C., Zhong, R., Zhang, J., Wang, X., Ding, G., Xiao, W., et al. (2019). Reduning Injection Ameliorates Paraquat-Induced Acute Lung Injury by Regulating AMPK/MAPK/NF-кB Signaling. J. Cel Biochem. 120, 12713-12723. doi: $10.1002 / j c b .28540$

Kong, Y., Wu, H. W., Chen, Y., Lai, S., Yang, Z. M., and Chen, J. S. (2020). Mechanism of Tanreqing Injection on Treatment of Coronavirus Disease 2019 Based on Network Pharmacology and Molecular Docking. Chin. Trad Herb Drugs 51, 1785-1793. doi:10.7501/j.issn.0253-2670.2020.07.012

Lai, X., Cao, K., Kong, L., Liu, Q., Gao, Y., and investigators, X. s. (2017). Xingnaojing for Moderate-To-Severe Acute Ischemic Stroke (XMAS): Study 
Protocol for a Randomized Controlled Trial. Trials 18, 479. doi:10.1186/ s13063-017-2222-y

Li, C., Wang, P., Zhang, L., Li, M., Lei, X., Liu, S., et al. (2018a). Efficacy and Safety of Xuebijing Injection (A Chinese Patent) for Sepsis: A Meta-Analysis of Randomized Controlled Trials. J. Ethnopharmacol 224, 512-521. doi:10.1016/ j.jep.2018.05.043

Li, C., Zang, C., Nie, Q., Yang, B., Zhang, B., and Duan, S. (2019a). Simultaneous Determination of Seven Flavonoids, Two Phenolic Acids and Two Cholesterines in Tanreqing Injection by UHPLC-MS/MS. J. Pharm. Biomed. Anal. 163, 105-112. doi:10.1016/j.jpba.2018.08.058

Li, H. R., Chang, L. P., Wei, C., and Jia, Z. H. (2020a). Theoretical Research Basis and Clinical Efficacy of Lianhua Qingwen in Treating Novel Coronavious Pneumonica. World Chin. Med. 15, 332-336. doi:10.3969/j.issn.16737202.2020 .03 .006

Li, P., Wang, T., Fu, Z., Chang, N., and Wang, X. (2016). Clinical Comparison of Shenfu Injection and Shenmai Injection Based on Meta Analysis. Chin. Trad Herb Drugs 47, 2949-2959. doi:10.7501/j.issn.0253-2670.2016.16.030

Li, Q., Li, Z. Y., Zhang, J., Guo, W. N., Xu, X. M., Sun, F. X., et al. (2019b). Xiyanping Plus Azithromycin Chemotherapy in Pediatric Patients with Mycoplasma Pneumoniae Pneumonia: A Systematic Review and MetaAnalysis of Efficacy and Safety. Evid. Based Complement. Alternat Med. 2019, 2346583. doi:10.1155/2019/2346583

Li, X., Shen, Y., He, F., Li, F., and Zhu, Y. (2018b). Effectiveness and Safety of Shenmai Injection in Treatment of Shock: A Meta-Analysis. J. Tradit Chin. Med. 38, 155-166. doi:10.1016/j.jtcm.2017.05.001

Li, X., Yang, Y., Liu, L., Yang, X., Zhao, X., Li, Y., et al. (2020). Effect of Combination Antiviral Therapy on Hematological Profiles in 151 Adults Hospitalized with Severe Coronavirus Disease 2019. Pharmacol. Res. 160, 105036. doi:10.1016/j.phrs.2020.105036

Li, X. H., Li, S. J., Xu, Y., Wei, D., Shi, Q. S., Zhu, Q. X., et al. (2017). Effect of Integrated Chinese and Western Medicine Therapy on Severe Hand, Foot and Mouth Disease: A Prospective, Randomized, Controlled Trial. Chin. J. Integr. Med. 23, 887-892. doi:10.1007/s11655-016-2504-3

Li, X. X., Zhuo, L., Zhang, Y., Yang, Y. H., Zhang, H., Zhan, S. Y., et al. (2019c). The Incidence and Risk Factors for Adverse Drug Reactions Related to Tanreqing Injection: A Large Population-Based Study in China. Front. Pharmacol. 10, 1523. doi:10.3389/fphar.2019.01523

Liang, W., Chen, T., and Liu, Q. (2019). Discussion on the Application of Angong Niuhuang Pill on Emergency Treatment. J. Emerg. Trad Chin. Med. 28, 483-486. doi:10.3969/j.issn.1004-745X.2019.03.029

Ling, X. Y., Tao, J., Sun, X., and Yuan, B. (2020). Exploring Material Basis and Mechanism of Lianhua Qingwen Prescription against Coronavirus Based on Network Pharmacology. Chin. Trad Herb Drugs 51, 1723-1730. doi:10.7501/ j.issn.0253-2670.2020.07.006

Liu, H., Yan, Y., Pang, P., Mao, J., Hu, X., Li, D., et al. (2019). Angong Niuhuang Pill as Adjuvant Therapy for Treating Acute Cerebral Infarction and Intracerebral Hemorrhage: A Meta-Analysis of Randomized Controlled Trials. J. Ethnopharmacol 237, 307-313. doi:10.1016/ j.jep.2019.03.043

Liu, S. S., and Xie, Y. M. (2017). Literature Analysis of 101 Cases of Adverse Reactions Induced by Huoxiangzhengqi Liquid. Chin. J. Pharmacovigil 14, 317-320. doi:10.3969/j.issn.1672-8629.2017.05.014

Liu, Z., Li, X., Gou, C., Li, L., Luo, X., Zhang, C., et al. (2020). Effect of Jinhua Qinggan Granules on Novel Coronavirus Pneumonia in Patients. J. Tradit Chin. Med. 40, 467-472. doi:10.19852/j.cnki.jtcm.2020.03.016

Luo, Z., Chen, W., Xiang, M., Wang, H., Xiao, W., Xu, C., et al. (2021). The Preventive Effect of Xuebijing Injection against Cytokine Storm for Severe Patients with COVID-19: A Prospective Randomized Controlled Trial. Eur. J. Integr. Med. 42, 101305. doi:10.1016/j.eujim.2021.101305

Lv, R. B., Wang, W., and Li, X. (2020). Combined with Western Medicine Conventional Therapy in the Treatment of 63 Suspected Cases of Coronavirus Disease 2019. J. Trad Chin. Med. 81, 655-659. doi:10.13288/ j.11-2166/r.2020.08.003

Ma, R., Ma, X., Wen, J., Wang, J., Xie, Q., Chen, N., et al. (2018). Preclinical Evidence and Mechanism of Xingnaojing Injection for Cerebral Ischemia: A Systematic Review and Meta-Analysis of Animal Studies. Evid. Based Complement. Alternat Med. 2018, 9624175. doi:10.1155/ 2018/9624175
Mao, Y., Su, Y. X., Xue, P., Li, L. L., and Zhu, S. J. (2020). Discussion on the Mechanism of Jinhua Qinggan Granules in the Treatment of Novel Coronavirus Pneumonia. J. Chin. Med. Mat 43, 2843-2849. doi:10.13863/ j.issn1001-4454.2020.11.046

Peng, S., Hang, N., Liu, W., Guo, W., Jiang, C., Yang, X., et al. (2016). Andrographolide Sulfonate Ameliorates Lipopolysaccharide-Induced Acute Lung Injury in Mice by Down-Regulating MAPK and NF-Kb Pathways. Acta Pharm. Sin B 6, 205-211. doi:10.1016/j.apsb.2016.02.002

Runfeng, L., Yunlong, H., Jicheng, H., Weiqi, P., Qinhai, M., Yongxia, S., et al. (2020b). Lianhuaqingwen Exerts Anti-viral and Anti-inflammatory Activity against Novel Coronavirus (SARS-CoV-2). Pharmacol. Res. 156, 104761. doi:10.1016/j.phrs.2020.104761

Shao, C. C., Wang, H. J., Meng, P. F., Chen, G. Z., Ma, X. X., Zhang, S. X., et al. (2020). TCM Understanding and Treatment Status of Covid-19. Acta Chin. Med. 35, 704-708. doi:10.16368/j.issn.1674-8999.2020.04.158

Shen, F., Fu, Z. Y., Wu, Y. R., Kuang, G. Y., Li, L., Zhu, K. M., et al. (2020). The Potential Targets and Mechanisms of Shufeng Jiedu Capsule for Novel Coronavirus Pneumonia (COVID-19) Based on Network Pharmacology and Molecular Docking. Guid J. Trad Chin. Med. Pharmacol. 26, 8-15. doi:10.13862/j.cnki.cn43-1446/r.2020.05.003

Shi, X., Wei, J., Liu, M. Y., Jin, X. H., Zhou, H. P., Zhu, W. L., et al. (2020). Study on the Overall Regulation of Xuebijing Injection in Treating COVID-19. Shanghai J. Trad Chin. Med. 54, 46-53. doi:10.16305/j.1007-1334.2020.04.098

Song, R., Dong, C., Wang, C., Zhang, H., and Yang, Z. (2018). [Effectiveness of Xuebijing in Treatment of Multiple Organ Dysfunction Syndrome: a Meta Analysis]. Zhonghua Wei Zhong Bing Ji Jiu Yi Xue 30, 848-854. doi:10.3760/ cma.j.issn.2095-4352.2018.09.006

Song, Y., Yao, C., Yao, Y., Han, H., Zhao, X., Yu, K., et al. (2019). XueBijing Injection versus Placebo for Critically Ill Patients with Severe CommunityAcquired Pneumonia: A Randomized Controlled Trial. Crit. Care Med. 47, e735-e743. doi:10.1097/CCM.0000000000003842

Su, H. X., Yao, S., Zhao, W. F., Li, M. J., Liu, J., Shang, W. J., et al. (2020). AntiSARS-CoV-2 Activities In Vitro of Shuanghuanglian Preparations and Bioactive Ingredients. Acta Pharmacol. Sin 41, 1167-1177. doi:10.1038/ s41401-020-0483-6

Sun, P. P., Zhang, N., and Liu, Y. Y. (2019). Study on Literature Analysis of Adverse Drug Reactions Caused by Reduning Injection. Prac Pharm. Clin. Rem 22, 190-193. doi:10.14053/j.cnki.ppcr.201902021

Tang, S. H., Li, D. M., Liao, Q., Qi, R. H., and Zhang, H. C. (2019). Meta-analysis of Clinical Efficacy of Tanreqing Injection for Community-Acquired Pneumonia. Chin. J. Clin. Res. 32, 294-301. doi:10.13429/j.cnki.cjcr.2019.03.002

Tao, Z., Gao, J., Zhang, G., Xue, M., Yang, W., Tong, C., et al. (2014). Shufeng Jiedu Capsule Protect against Acute Lung Injury by Suppressing the MAPK/NF- $\mathrm{B}$ Pathway. Biosci. Trends 8, 45-51. doi:10.5582/bst.8.45

The Lancet (2020). Emerging Understandings of 2019-nCoV. Lancet 395, 311. doi:10.1016/S0140-6736(20)30186-0

Wang, C., Shi, Q. P., Ding, F., Jiang, X. D., Tang, W., Yu, M. L., et al. (2019a). Reevaluation of the post-marketing Safety of Xuebijing Injection Based on RealWorld and Evidence-Based Evaluations. Biomed. Pharmacother. 109, 1523-1531. doi:10.1016/j.biopha.2018.10.190

Wang, Y. G., Qi, W. S., Ma, J. J., Ruan, L. G., Lu, Y. R. C. L. X., et al. (2020). Clinical Features and Syndrome Differentiation of Novel Coronavirus Pneumonia in Traditional Chinese Medicine. J. Trad Chin. Med. 61, 281-285. doi:10.13288/ j.11-2166/r.2020.04.002

Wang, Y. M., Lv, L., Fan, H. W., and Xu, H. F. (2018). Meta-analysis on Effectiveness and Safety of Xiyanping Injection Combined with Antibiotics in Treatment of CAP in Adults. Chin. Trad Herb Drugs 49, 468-476. doi:10.7501/j.issn.0253-2670.2018.02.031

Wang, Z. F., Zhang, H. C., Xie, Y. M., Miao, Q., Sun, Z. T., Wang, R. B., et al. (2019b). [Expert Consensus Statement on Xiyanping Injection for Respiratory System Infectious Diseases in Clinical Practice(adults)]. Zhongguo Zhong Yao Za Zhi 44, 5282-5286. doi:10.19540/j.cnki.cjcmm.20191105.502

Wen, L., Zhou, Z., Jiang, D., and Huang, K. (2020). [Effect of Xuebijing Injection on Inflammatory Markers and Disease Outcome of Coronavirus Disease 2019]. Zhonghua Wei Zhong Bing Ji Jiu Yi Xue 32, 426-429. doi:10.3760/ cma.j.cn121430-20200406-00386

Wu, L., Zhang, H., Xing, Y., Gao, Y., Li, Y., Ren, X., et al. (2016). MetaAnalysis of the Effects of Xingnaojing Injection on Consciousness 
Disturbance. Medicine (Baltimore) 95, e2875 doi:10.1097/ MD.0000000000002875

Xia, Q. D., Xun, Y., Lu, J. L., Lu, Y. C., Yang, Y. Y., Zhou, P., et al. (2020). Network Pharmacology and Molecular Docking Analyses on Lianhua Qingwen Capsule Indicate Akt1 Is a Potential Target to Treat and Prevent COVID-19. Cell Prolif 53, e12949. doi:10.1111/cpr.12949

Xiang, D., Huang, Y., and Deng, L. (2017). Analysis of 1533 Cases of Adverse Drug Reactions/events in Clinical Practice Caused by Shenmai Injection. Chin. J. Hosp. Pharm. 37, 393-396. doi:10.1097/psn.0000000000000200

Xiao, M., Tian, J., Zhou, Y., Xu, X., Min, X., Lv, Y., et al. (2020). Efficacy of Huoxiang Zhengqi Dropping Pills and Lianhua Qingwen Granules in Treatment of COVID-19: A Randomized Controlled Trial. Pharmacol. Res. 161, 105126. doi:10.1016/j.phrs.2020.105126

Xu, X., Zhang, J., Zheng, W., Yang, Z., Zhao, X., Wang, C., et al. (2021). Efficacy and Safety of Reduning Injection in the Treatment of COVID-19: a Randomized, Multicenter Clinical Study. Ann. Palliat. Med. 10, 5146-5155. doi:10.21037/ apm-20-2121

Xu, Y. F., Chen, G., and Xu, C. (2015). Influence of Andrographolide on Expression of TLR4, CD14, MD2 mRNA in Acute Lung Injury in Rats Induced by Lipopolysaccharide. Chin. Arch. Trad Chin. Med. 33, 1198-1200. doi:10.13193/j.issn.1673-7717.2015.05.054

Yan, B. H., Jiang, Z. W., Zeng, J. P., Tang, J. Y., Ding, H., Xia, J. L., et al. (2020). [Large- Scale Prospective Clinical Study on Prophylactic Intervention of COVID-19 in Community Population Using Huoxiang Zhengqi Oral Liquid and Jinhao Jiere Granules]. Zhongguo Zhong Yao Za Zhi 45, 2993-3000. doi:10.19540/j.cnki.cjcmm.20200430.501

Yang, W., Liu, J., Blažeković, B., Sun, Y., Ma, S., Ren, C., et al. (2018). In Vitro antibacterial Effects of Tanreqing Injection Combined with Vancomycin or Linezolid against Methicillin-Resistant Staphylococcus aureus. BMC Complement. Altern. Med. 18, 169. doi:10.1186/s12906-018-2231-8

Ye, M., Luo, G., Ye, D., She, M., Sun, N., Lu, Y. J., et al. (2020). Network Pharmacology, Molecular Docking Integrated Surface Plasmon Resonance Technology Reveals the Mechanism of Toujie Quwen Granules against Coronavirus Disease 2019 Pneumonia. Phytomedicine, 153401. doi:10.1016/ j.phymed.2020.153401

Yu, H., Ren, X., Qi, X., Zuo, Q., and Liu, D. (2020). Efficacy Study of Arbidol, Qingfei Paidu Decoction, Lianhuaqingwen Capsule, and Jinye Baidu Granules in the Treatment of Mild/Moderate COVID-19 in a Fangcang Shelter Hospital. Pharmacol. Clin. Chin. Mat Med. 36 (6), 2-6. doi:10.13412/j.cnki.zyyl.20201110.005

Zhang, G., Hou, Y., Li, Y., He, L., Tang, L., Yang, T., et al. (2017a). Xiyanping Injection Therapy for Children with Mild Hand Foot and Mouth Disease: a Randomized Controlled Trial. J. Tradit Chin. Med. 37, 397-403. Available at: https://www.ncbi.nlm.nih.gov/pubmed/31682384.
Zhang, G., Zhao, J., He, L., Yan, S., Zhuo, Z., Zheng, H., et al. (2013). Reduning Injection for Fever, Rash, and Ulcers in Children with Mild Hand, Foot, and Mouth Disease: a Randomized Controlled Clinical Study. J. Tradit Chin. Med. 33, 733-742. doi:10.1016/s0254-6272(14)60005-4

Zhang, S., Zhao, H., and Luo, Y. (2017b). The Clinical Application of Xingnaojing Injection in Neurological Diseases. Chin. J. Integ Med. Cardio-/cerebrovas Dis. 15, 2720-2723. doi:10.3969/j.issn.1672-1349.2017.21.017

Zhang, T., Zhu, Y. X., Liu, D. L., Bai, G., Xu, J., and Hang, Y. Q. (2016). Study on Pharmacodynamic Material Basis and Mechanism of Shufeng Jiedu Capsule. Chin. Trad Herb Drugs 47, 2019-2026. doi:10.7501/j.issn.0253-2670.2016.12.001

Zhang, X., Xue, Y., Chen, X., Wu, J.-m., Su, Z.-j., Sun, M., et al. (2021a). Effects of Tanreqing Capsule on the Negative Conversion Time of Nucleic Acid in Patients with COVID-19: A Retrospective Cohort Study. J. Integr. Med. 19, 36-41. doi:10.1016/j.joim.2020.10.002

Zhang, Y. L., Zhao, W., Jin, M. Y., Gui, Y., Zhang, Q., Gong, Z. L., et al. (2020b). Pharmaceutical Care of Traditional Chinese Medicine in Preventing and Treating the Coronavirus Disease 2019. China Pharm. 29, 27-34. doi:10.3969/j.issn.1006-4931.2020.05.006

Zhao, J., Tian, S., Lu, D., Yang, J., Zeng, H., Zhang, F., et al. (2020). Systems Pharmacological Study Illustrates the Immune Regulation, Anti-infection, Anti-inflammation, and Multi-Organ protection Mechanism of Qing-FeiPai-Du Decoction in the Treatment of COVID-19. Phytomedicine 85, 153315. doi:10.1016/j.phymed.2020.153315

Zhao, L., Peng, F., Guan, B., Li, X., Wu, W., Chen, J., et al. (2015). Whether Metal Element-Containing Herbal Formula Angong Niuhuang Pill Is Safe for Acute Brain Disorders?. Biol. Trace Elem. Res. 166, 41-48. doi:10.1007/s12011-015-0318-3

Conflict of Interest: The authors declare that the research was conducted in the absence of any commercial or financial relationships that could be construed as a potential conflict of interest.

Publisher's Note: All claims expressed in this article are solely those of the authors and do not necessarily represent those of their affiliated organizations, or those of the publisher, the editors, and the reviewers. Any product that may be evaluated in this article, or claim that may be made by its manufacturer, is not guaranteed or endorsed by the publisher.

Copyright (C) $2021 \mathrm{Wu}$ and Zhong. This is an open-access article distributed under the terms of the Creative Commons Attribution License (CC BY). The use, distribution or reproduction in other forums is permitted, provided the original author(s) and the copyright owner(s) are credited and that the original publication in this journal is cited, in accordance with accepted academic practice. No use, distribution or reproduction is permitted which does not comply with these terms. 